\title{
AN EXTENSION OF THE FEJÉR-JACKSON INEQUALITY
}

\author{
GAVIN BROWN and KUN-YANG WANG
}

(Received 1 December 1992; revised 9 February 1994)

Communicated by P. G. Dodds

\begin{abstract}
Best-possible results are established for positivity of the partial sums of $\sum \sin k \theta(k+\alpha)^{-1}$. In fact odd sums are positive for $-1 \leq \alpha \leq \alpha_{0}=2.1 \ldots$, while sums with $2 k$ terms are positive on the subinterval $] 0, \pi-2 \mu_{0} \pi(4 k+1)^{-1}\left[, \mu_{0}=0.8128 \ldots\right.$. This is analagous to the Gasper extension of the Szegö-Rogosinski-Young inequality for cosine sums.
\end{abstract}

1991 Mathematics subject classification (Amer. Math. Soc.): primary 42A05; secondary 42A10, 42A16.

\section{Introduction}

It is, of course, well-known that, for every positive integer $n$,

$$
\sum_{k=1}^{n} \frac{\sin k \theta}{k}>0, \quad 0<\theta<\pi
$$

This inequality was first mentioned by Fejér in 1910 (see [3]), was proved by Jackson, and subsequently revisited by many mathematicians who have offered different proofs. In parallel, for cosine series, Young [6] proved the following inequality:

$$
1+\sum_{k=1}^{n} \frac{\cos k \theta}{k} \geq 0, \quad 0 \leq \theta \leq \pi .
$$

Rogosinski and Szegö [5] extended Young's inequality to

$$
\frac{1}{1+\alpha}+\sum_{k=1}^{n} \frac{\cos k \theta}{k+\alpha} \geq 0, \quad 0 \leq \theta \leq \pi, \quad-1<\alpha \leq 1 .
$$

(C) 1997 Australian Mathematical Society 0263-6115/97 \$A2.00+0.00 
The case $\alpha=1$ is interesting but Gasper showed in [4] that the result admits considerable improvement. In fact he showed that (3) holds for $-1<\alpha \leq \tilde{\alpha}=4.567 \ldots$, and this $\tilde{\alpha}$ is best possible.

In the present paper, we will extend the Fejér-Jackson inequality in a similar way. Thus we shall be concerned with the partial sums

$$
T_{n}^{\alpha}(\theta)=\sum_{k=1}^{n} \frac{\sin k \theta}{k+\alpha} \quad(\alpha>-1, n \in \mathbb{N}) .
$$

The odd partial sums are positive for $-1<\alpha \leq \alpha_{0}$, where $\alpha_{0}=2.1 \ldots$ is best possible. The result for even partial sums holds only on a subinterval $] 0, \pi-2 \mu_{0} \pi(4 n+$ $1)^{-1}$ [, where $\mu_{0}=0.8128252 \ldots$ is best possible.

To make matters precise we must define three constants $\lambda_{0}, \mu_{0}, \alpha_{0}$. The first of these is the solution of the equation

$$
(1+\lambda) \pi=\tan (\lambda \pi) \quad 0<\lambda<1 / 2,
$$

and it is easy to see that $\lambda_{0}=0.4302967 \ldots$ is the point at which the function $(\sin \lambda \pi) /(1+\lambda)$ attains its maximum for $0<\lambda<1 / 2$. We define $\mu_{0}$ to be the solution of

$$
\frac{\sin \mu \pi}{\mu \pi}=\frac{\sin \lambda_{0} \pi}{\left(1+\lambda_{0}\right) \pi}
$$

and $\alpha_{0}$ to be the solution of the equation

$$
\sum_{k=1}^{\infty} \frac{2 k}{(2 k-1+\alpha)(2 k+\alpha)(2 k+1+\alpha)}=\frac{\sin \lambda_{0} \pi}{2\left(1+\lambda_{0}\right) \pi} .
$$

The main results can now be stated.

THEOREM 1. If $-1<\alpha \leq \alpha_{0}$ then

$$
T_{2 n-1}^{\alpha}(\theta)>0, \quad 0<\theta<\pi, \quad n \in \mathbb{N} .
$$

THEOREM 2. If $-1<\alpha \leq \alpha_{0}$ then

$$
T_{2 n}^{\alpha}(\theta)>0, \quad 0<\theta \leq \pi-\mu_{0} \pi /(2 n+0.5) .
$$

THEOREM 3. If $\alpha>\alpha_{0}$ then there exists an infinite subset $N \subset \mathbb{N}$ such that

$$
T_{2 n-1}^{\alpha}\left(\pi-\frac{\left(1+\lambda_{0}\right) \pi}{2 n-\frac{1}{2}}\right)<0, \quad n \in N
$$


THEOREM 4. If $0<\gamma<\mu_{0}$ then there exists an $\alpha$ near to but strictly smaller than $\alpha_{0}$ such that

$$
T_{2 n}^{\alpha}(\pi-\gamma \pi /(2 n+0.5))<0
$$

for an infinite number of $n$.

From Theorem 3 we see that $\alpha_{0}$ is best possible in Theorem 1. Theorem 4 shows that $\mu_{0}$ is best possible in Theorem 2 .

The particular case $\alpha=1$ has been considered by Brown and Wilson [2]. They obtained the following conclusion:

$$
T_{2 n-1}^{1}(\theta)>0, \quad 0<\theta<\pi ; \quad T_{2 n}^{1}(\theta)>0, \quad 0<\theta<\pi-\pi / 2 n .
$$

\section{Basic lemmas}

LEMMA 1. For $n \geq 3, \alpha>-1$, double partial summation gives

$$
T_{n}^{\alpha}(\theta)=\sum_{k=1}^{n-2} a_{k}(\alpha) \sigma_{k}(\theta)+\frac{\sigma_{n-1}(\theta)}{(n-1+\alpha)(n+\alpha)}+\frac{S_{n}(\theta)}{n+\alpha}
$$

where

$$
a_{k}(\alpha)=\frac{2}{(k+\alpha)(k+1+\alpha)(k+2+\alpha)}, \quad S_{k}(\theta)=\sum_{j=1}^{k} \sin j \theta, \quad \sigma_{k}(\theta)=\sum_{J=1}^{k} S_{j}(\theta) .
$$

LEMMA 2. For $0<\theta<\pi$,

$$
\sigma_{k}(\theta)>0 \text {. }
$$

LEMMA 3. Let $0<\delta<2, t_{n}=\delta \pi /\left(n+\frac{1}{2}\right)$ and $\theta_{n}=\pi-t_{n}$. Then

$$
\lim _{n \rightarrow \infty} \frac{\sigma_{k}\left(\theta_{n}\right)}{\sin \theta_{n}}= \begin{cases}(k+1) / 2 & \text { if } k \text { is odd, } \\ 0 & \text { if } k \text { is even },\end{cases}
$$

and

$$
0<\frac{\sigma_{k}\left(\theta_{n}\right)}{\sin \theta_{n}}<k+1, \quad 1 \leq k \leq n, \quad n \geq 7 .
$$

All these lemmas admit simple direct proofs. We learned of (6) from [1, (1.9) p.8]. The inequality was found by Lukacs and published in Fejér's paper [3]. 


\section{Proof of Theorems 3 and 4}

ProOF OF THEOREM 3. Here we write $\theta_{n}=\pi-\left(1+\lambda_{0}\right) \pi /\left(2 n-\frac{1}{2}\right)$. Suppose $T_{2 n-1}^{\alpha}\left(\theta_{n}\right)>0$ for all sufficiently large $n \in \mathbb{N}$. Then by (5) we get

$$
\sum_{k=1}^{2 n-3} a_{k}(\alpha) \frac{\sigma_{k}\left(\theta_{n}\right)}{\sin \theta_{n}}+\frac{1}{(2 n-2+\alpha)(2 n-1+\alpha)} \frac{\sigma_{2 n-2}\left(\theta_{n}\right)}{\sin \theta_{n}}>-\frac{1}{2 n-1+\alpha} \frac{S_{2 n-1}\left(\theta_{n}\right)}{\sin \theta_{n}}
$$

and the right hand side of (9) tends to $\left(\sin \lambda_{0} \pi\right) / 2\left(1+\lambda_{0}\right) \pi$ as $n$ tends to infinity.

By Lemma 3 and using the Dominated Convergence Theorem we deduce from (9) that

$$
\sum_{k=1}^{\infty} k a_{2 k-1}(\alpha) \geq \frac{\sin \lambda_{0} \pi}{2\left(1+\lambda_{0}\right) \pi}
$$

Noticing that $k a_{2 k-1}(\alpha)$ is decreasing when $\alpha$ is increasing, we conclude that (10) is equivalent to $\alpha \leq \alpha_{0}$. This completes the proof.

PROOF OF THEOREM 4. Write $u_{n}=\gamma \pi /\left(2 n+\frac{1}{2}\right), 0<\gamma<\mu_{0}$. For $n \geq 2$ we have

$$
\begin{aligned}
& \frac{1}{\sin u_{n}} T_{2 n}^{\alpha}\left(\pi-u_{n}\right) \\
& \quad=\sum_{k=1}^{2 n-2} a_{k}(\alpha) \frac{\sigma_{k}\left(\pi-u_{n}\right)}{\sin u_{n}}+\frac{1}{(2 n-1+\alpha)} \frac{\sigma_{2 n-1}\left(\pi-u_{n}\right)}{\sin u_{n}}+\frac{1}{2 n+\alpha} \frac{S_{2 n}\left(\pi-u_{n}\right)}{\sin u_{n}} .
\end{aligned}
$$

Let us write $F(\alpha)=\sum_{k=1}^{\infty} k a_{2 k-1}(\alpha)$. Then by Lemma 3 and the Dominated Convergence Theorem we get

$$
\lim _{n \rightarrow \infty} \frac{1}{\sin u_{n}} T_{2 n}^{\alpha}\left(\pi-u_{n}\right)=F(\alpha)-\frac{\sin \gamma \pi}{2 \gamma \pi} .
$$

As we chose $F\left(\alpha_{0}\right)=\left(\sin \mu_{0} \pi\right) / 2 \mu_{0} \pi$, it follows that $T_{2 n}^{\alpha}\left(\pi-u_{n}\right)$ is negative for $\alpha$ near to but strictly less than $\alpha_{0}$ and $n$ big enough.

\section{Proof of Theorem 1}

We achieve the proof by developing a sequence of lemmas. 
LEMMA 4. Let

$$
-\frac{d}{d \alpha} T_{n}^{\alpha}(\theta)=U_{n}^{\alpha}(\theta)=\sum_{k=1}^{n} \frac{\sin k \theta}{(k+\alpha)^{2}}, \quad \alpha>-1, \quad n \in \mathbb{N} .
$$

If $-1<\alpha \leq 2.5$ then

$$
U_{2 n-1}^{\alpha}(\theta)>0, \quad 0<\theta<\pi, \quad n \in \mathbb{N} .
$$

PROOF. Simple direct estimates for the other cases allow us to assume $n \geq 3$ and $1<\alpha \leq 2.5$. By using partial summation twice we get

$$
U_{2 n-1}^{\alpha}(\theta)=\sum_{k=1}^{2 n-3} b_{k}(\alpha) \sigma_{k}(\theta)+\left(c_{2 n-2}(\alpha)-c_{2 n-1}(\alpha)\right) \sigma_{2 n-2}(\theta)+c_{2 n-1}(\alpha) S_{2 n-1}(\theta),
$$

where $b_{k}(\alpha)=c_{k}(\alpha)-2 c_{k+1}(\alpha)+c_{k+2}(\alpha), c_{k}(\alpha)=(k+\alpha)^{-2}$.

Noticing

$$
S_{2 n-1}(\theta)=\frac{\cos \theta / 2-\cos \left(2 n-\frac{1}{2}\right) \theta}{2 \sin \theta / 2} \geq-\frac{1}{2} \tan \theta / 4
$$

we get

$$
2 \cot \frac{\theta}{4} \cdot U_{2 n-1}^{\alpha}(\theta)>\left(b_{1}(\alpha)+b_{3}(\alpha)\right) 8 \cos ^{2} \frac{\theta}{4} \cos \frac{\theta}{2}-\frac{1}{(2 n-1+\alpha)^{2}} \quad(n \geq 3) .
$$

If $0<\theta \leq 0.85 \pi$ and $1 \leq \alpha \leq 2$ then

$$
2 \cot \frac{\theta}{4} U_{2 n-1}^{\alpha}(\theta)>\left(b_{1}(2)+b_{3}(2)\right) \times 1.15177-\frac{1}{36}>0 \quad(n \geq 3) .
$$

When $2<\alpha \leq 2.5$ and $0<\theta \leq 0.85 \pi$, since

$$
b_{1}(\alpha)+b_{3}(\alpha) \geq b_{1}(2.5)+b_{3}(2.5)=0.01942
$$

we have

$$
2 \cot \frac{\theta}{4} U_{2 n-1}^{\alpha}(\theta)>0.01942 \times 1.15177-\frac{1}{49}>0 \quad(n \geq 3) .
$$

On the other hand, for $0.85 \pi<\theta<\pi$, we set $t=\pi-\theta$. Then

$$
U_{2 n-1}^{\alpha}(\theta) \geq\left(b_{1}(\alpha)+b_{3}(\alpha)\right) \sin t+\frac{1}{(2 n-1+\alpha)^{2}} \frac{\sin t / 2+\sin \left(2 n-\frac{1}{2}\right) t}{2 \cos t / 2} .
$$

We see, if $t \leq \pi /\left(2 n-\frac{1}{2}\right)$, the above sum is strictly positive. Assume $\pi /\left(2 n-\frac{1}{2}\right)<$ $t<0.15 \pi$. This occurs only when $n \geq 4$. Then we have

$$
\frac{1}{t} U_{2 n-1}^{\alpha}(\pi-t)>\left(b_{1}(\alpha)+b_{3}(\alpha)\right) \frac{\sin 0.15 \pi}{0.15 \pi}-\frac{0.51421\left(2 n-\frac{1}{2}\right)}{(2 n-1+\alpha)^{2} \pi}
$$

and we estimate for $1 \leq \alpha \leq 2,2 \leq \alpha \leq 2.5$ as before. 
LEMMA 5. Let

$$
\begin{aligned}
f(\theta) & =a_{1}(2.11) \sigma_{1}(\theta)+a_{2}(2.11) \sigma_{2}(\theta)+a_{3}(2.11) \sigma_{3}(\theta) \\
g_{n}(\theta) & =\frac{\sigma_{2 n-2}(\theta)}{2 n+0.11}+S_{2 n-1}(\theta) \quad(n \geq 3) .
\end{aligned}
$$

If $0<\theta \leq 0.75 \pi$ and $n \geq 3$ then

$$
T_{2 n-1}^{\alpha_{0}}(\theta)>f(\theta)+\frac{1}{2 n+1.11} g_{n}(\theta)>0 .
$$

PROOF. By Lemma 4 we have $T_{2 n-1}^{\alpha_{0}}(\theta)>T_{2 n-1}^{2.11}(\theta)$. Then using Lemma 1 we get for $n \geq 3$

$$
T_{2 n-1}^{\alpha_{0}}(\theta)>T_{2 n-1}^{2.11}(\theta) \geq f(\theta)+\frac{1}{2 n+1.11} g_{n}(\theta)
$$

It is easy to establish that $0.0503 \sin \theta$ is a lower bound for $f(\theta)$, for $0<\theta \leq 0.75 \pi$. For $0<\theta<0.5 \pi$, this can be combined with the lower bound $-0.5 \tan \theta / 4$ for $g_{n}(\theta)$ to establish the result. For $0.5 \pi<\theta \leq 0.75 \pi$, we use the estimate

$$
g_{n}(\theta)>\left[\frac{2 n-1-\operatorname{cosec} \theta}{(2 n+0.11) 4 \sin ^{2} \theta / 2}-\frac{\sin ^{2} \theta / 4}{\sin \theta / 2-\sin \theta}\right] \sin \theta>-0.3006 \sin \theta .
$$

The next two lemmas are straightforward estimates based on Lemma 1.

LEMMA 6. Let $t=(1+\delta) \pi / 5.5,0<\delta \leq 0.375$. Then $T_{5}^{\alpha_{0}}(\pi-t)>0$.

LEMMA 7. Let $t=(2 \ell-1+\delta) \pi /(2 n-0.5), \ell \in \mathbb{N}, 0<\delta<1, n \geq 4$. Define

$$
U_{n}(t)=\frac{\cos ^{2} t / 2 \cdot T_{2 n-1}^{\alpha_{0}}(\pi-t)}{\sin t},
$$

$D_{n}(t)=\sum_{k=1}^{\infty} k a_{2 k-1}\left(\alpha_{0}\right)-\frac{\sin \delta \pi}{2(2 \ell-1+\delta) \pi} u_{n}(t)-\frac{1}{2} \sum_{k=1}^{n-2} a_{2 k+1} \frac{\sin ^{2}\left(k+\frac{3}{4}\right) t}{\cos t / 2}-v_{n}(t)$

with

$u_{n}(t)=\left(1-\frac{\alpha_{0}-0.5}{2 n-1+\alpha_{0}}\right) \frac{t}{2 \sin t / 2}, \quad v_{n}(t)=\frac{\left(\alpha_{0}-1\right) \sin t+\sin (2 n-1) t}{\left(2 n-2+\alpha_{0}\right)\left(2 n-1+\alpha_{0}\right) 4 \sin t}$,

and

$$
\Delta_{n}(t)=\frac{\alpha_{0}}{2} \sum_{k=n}^{\infty} a_{2 k-1}+\frac{\sin ^{2} t / 4}{2 \cos t / 2} \sum_{k=1}^{n-2} a_{2 k+1}-\sin ^{2} \frac{t}{2} a_{1},
$$

where $a_{k}=a_{k}\left(\alpha_{0}\right)$. Then

$$
U_{n}(t)>\Delta_{n}(t)+D_{n}(t)
$$


LEMMA 8. If $n \geq 4,0<\delta \leq 0.55$ and $t=(1+\delta) \pi /(2 n-0.5)$ then

$$
D_{n}(t)>0
$$

PROOF. By definition of $D_{n}$ and the fact that $F\left(\alpha_{0}\right) \geq(\sin \delta \pi) / 2(1+\delta) \pi$ we get

$$
D_{n}(t) \cos \frac{t}{2}>F\left(\alpha_{0}\right)\left(1-u_{n}(t)\right) \cos \frac{t}{2}-v_{n}(t)-\frac{1}{2} \sum_{k=1}^{n-2} a_{2 k+1} \sin ^{2}\left(k+\frac{3}{4}\right) t
$$

Simple power series estimates yield

$$
\begin{aligned}
u_{n}(t) & <1-\frac{1.6}{2 n+1.1}+\frac{0.9880}{(2 n-0.05)^{2}-0.9880} \\
v_{n}(t) & <\frac{0.4053}{(2 n+0.1)(2 n+1.1)}, \quad 0<\delta<1 \\
\cos \frac{t}{2} & \geq 1-\frac{2.9640}{(2 n-0.5)^{2}} .
\end{aligned}
$$

Combining these inequalities with (crude) direct estimates of the upper bound of $0.5 \sum_{k=1}^{n-2} a_{2 k+1} \sin ^{2}(k+3 / 4) t$ for each $n$ from 4 to 12 allows us to verify that $D_{n}(t)>$ 0 for $4 \leq n \leq 12$.

Now we assume $n \geq 13$ and observe that

$$
\begin{aligned}
(2 n- & 0.5)^{2} D_{n}(t) \cos \frac{t}{2} \\
> & F\left(\alpha_{0}\right)\left(3.2 n-4.3640-\frac{0.5950}{2 n+1.11}-\frac{0.9761}{(2 n-0.5)^{2}-0.9880}\right) \\
& \quad-\frac{(1.55 \pi)^{2}}{8} \sum_{k=1}^{n-2} \frac{1}{k+4.65}-0.4053 \\
& >0.3476 n-0.8821-2.9640 \log ((n+2.65) / 4.65) .
\end{aligned}
$$

If we write

$$
\phi(n)=0.3476 n-0.8821-2.9640 \log ((n+2.65) / 4.65)
$$

then we find

$$
\phi(13)=0.0396>0, \quad d \phi / d n>0 \text { for } n \geq 13
$$

Hence we conclude $D_{n}(t)>0$ for $n \geq 13$ and complete the proof.

LEMMA 9. If $n \geq 4,0.55<\delta<1$ and $t=(1+\delta) \pi /(2 n-0.5)$ then $D_{n}(t)>0$. 
PROOF. It is easy to verify that

$$
\sin \left(\delta \pi-\frac{0.5(1+\delta) \pi}{2 n-0.5}\right)>\left(\alpha_{0}-1\right) \sin \frac{(1+\delta) \pi}{2 n-0.5}
$$

for $0.55<\delta \leq 0.8$ and $n \geq 4$. Hence by (13), $-v_{n}(t)>0(0.55<\delta \leq 0.8, n \geq 4)$.

On the other hand,

$$
\frac{\sin \delta \pi}{2(1+\delta) \pi} \leq \frac{\sin 0.55 \pi}{2(1.55 \pi)}=0.10142 \quad(\delta>0.55)
$$

Therefore

$$
D_{n}(t) \geq 0.10862-0.10142 u_{n}(t)-\frac{1}{2 \cos t / 2} \sum_{k=1}^{n-2} a_{2 k+1} \sin ^{2}\left(k+\frac{3}{4}\right) t
$$

when $0.55<\delta \leq 0.8$ and $n \geq 4$. In this case,

$$
\begin{aligned}
u_{n}(t) & <\left(1-\frac{1.6}{2 n+1.11}\right)\left(1+\frac{(1.8 \pi)^{2}}{24(2 n-0.5)^{2}-(1.8 \pi)^{2}}\right) \\
& <1-\frac{1.6}{2 n+1.11}+\frac{1.3324}{(2 n-0.5)^{2}-1.3324} .
\end{aligned}
$$

According to (15) and (16), calculating directly we get

$$
\begin{aligned}
D_{4}(t) \geq & 0.00720+\frac{0.16227}{(2 n+1.11)}-\frac{0.13513}{(2 n-0.5)^{2}-1.3324} \\
& -0.53776\left(\alpha_{3} \sin ^{2} \frac{7}{4} t+a_{5}\right)>0.01592 \\
D_{5}(t) \geq & 0.02029-0.52999\left(a_{3} \sin ^{2} \frac{7}{4} t+a_{5}+a_{7}\right)>0.01374 \\
D_{6}(t) \geq & 0.01854-0.51550\left(a_{3} \sin ^{2}\left(\frac{7}{4} \frac{1.8 \pi}{11.5}\right)+a_{5}+a_{7}+a_{9}\right)>0.01229 .
\end{aligned}
$$

For $n \geq 7$ we have

$$
\begin{aligned}
& (2 n-0.5)^{2} D_{n}(t) \\
& \quad>0.00720(2 n-0.5)^{2}+0.15099(2 n-2.11)-4.08649 \log ((n+2.65) / 4.65) .
\end{aligned}
$$

It is easy to see this is positive by an argument similar to that in the proof of Lemma 8.

Assume now $0.8<\delta<1$. In this case

$$
\frac{\sin \delta \pi}{2(1+\delta) \pi}<0.05197, \quad u_{n}<1, \quad v_{n}<0.00207 .
$$


Hence

$$
D_{n}(t)>0.05458-5.40181 \frac{1}{(2 n-0.5)^{2}} \log \frac{n+2.65}{4.65}>0 .
$$

LEMMA 10. If $t=(1+\delta) \pi /(2 n-0.5), 0<\delta<1$ and $n \geq 4$, then $\Delta_{n}(t)>0$.

ProOF. Since $2.10<\alpha_{0}<2.11$, we have

$$
\frac{1}{2} \alpha_{0} \sum_{k=n}^{\infty} a_{2 k-1}>2.10 \sum_{k=n}^{\infty}(2 k+2.11)^{-3} .
$$

It turns out that the ensuing estimate

$$
\Delta_{n}(t)>0.13125(n+1.055)^{-3}+\sin ^{2} \frac{t}{4} \frac{a_{3}+a_{5}}{2}-a_{2} \sin ^{2} \frac{t}{2}
$$

leads quickly to the required result.

Lemmas 8-10 treat the case $\ell=1$. Now we assume $\ell \geq 2, t=(2 \ell-1+\delta) \pi /(2 n-$ $0.5), 0<\delta<1$. And we keep $t<0.25 \pi$. This restriction requires $n \geq 7$.

LEMMA 11. If $t=(2 \ell-1+\delta) \pi /(2 n-0.5)<0.25 \pi, 0<\delta<1$, and $\ell \geq 2$ then $T_{2 n-1}^{\alpha_{0}}(\pi-t)>0$.

PRoOF. In this case $u(t)<\pi(8 \sin \pi / 8)^{-1}$, [ $\left.\sin \delta \pi / 2(2 \ell-1+\delta) \pi\right]<(6 \pi)^{-1}$, $v_{n}(t)<0.00197(n \geq 7)$ and

$$
\frac{1}{2} \sec \frac{t}{2} \sum_{k=1}^{n-2} a_{2 k+1} \sin ^{2}\left(k+\frac{3}{4}\right) t<\frac{1}{2} \sec \frac{\pi}{8} \sum_{2}^{\infty} a_{2 k-1} .
$$

These estimates combined show that $D_{n}(t)>a_{1}$, and since $\Delta_{n}(t)>-a_{1}$, we achieve the proof.

Lemmas 5-11 now demonstrate that $T_{2 n-1}^{\alpha_{0}}(\theta)>0,0<\theta<\pi$, for $n \geq 3$. We complete the proof of Theorem 1 by checking the case $n=2$ directly.

\section{Proof of Theorem 2}

Because of the many parallels with the proof of Theorem 1 we merely sketch the salient steps. A fuller version of this (and the preceding proofs) is available from the authors on request. 
LEMMA 12. For $U_{n}^{\alpha}$ as in Lemma 4, if $-1<\alpha \leq 2.3$ then $U_{2 n}^{\alpha}(\theta)>0,0<\theta \leq$ $\pi-\mu_{0} \pi /(2 n+0.5)($ recall $\mu \approx 0.8128252)$.

ProOf. We have, in a way similar to the proof of Lemma 4,

$$
2 \cot \frac{\theta}{4} U_{2 n}^{\alpha}(\theta)>\left(b_{1}(\alpha)+b_{3}(\alpha)\right) 8 \cos ^{2} \frac{\theta}{4} \cos \frac{\theta}{2}-\frac{1}{(2 n+\alpha)^{2}} \quad(n \geq 3) .
$$

So, we get $U_{2 n}^{\alpha}(\theta)>0$ for $0<\theta \leq 0.85 \pi$ and $-1<\alpha \leq 2.3, n \geq 3$. On the other hand, when $0.85 \pi<\theta \leq \pi-\mu_{0} \pi /(2 n+0.5)$ by writing $t=\pi-\theta$ we have

$$
U_{2 n}^{\alpha}(\theta)>\left(b_{1}(\alpha)+b_{3}(\alpha)\right) \sin t+\frac{1}{(2 n+\alpha)^{2}} \frac{\sin \frac{t}{2}-\sin (2 n+0.5) t}{2 \cos \frac{t}{2}},
$$

and this leads quickly to a proof. The cases $n=1,2$ are handled separately.

Now to establish Theorem 4 we need only prove

$$
T_{2 n}^{\alpha_{0}}(\theta)>0, \quad 0<\theta \leq\left(1-\frac{\mu_{0}}{2 n+0.5}\right) \pi, \quad n \in \mathbb{N} .
$$

By an argument similar to Lemma 5 , we get

LEMMA 13. If $0<\theta \leq 0.75 \pi$ and $n \geq 3$ then $T_{2 n}^{\alpha_{0}}(\theta)>0$.

Now we assume $\theta=\pi-t, \mu_{0} \pi /(2 n+0.5) \leq t<0.25 \pi$ and write $V_{n}(t)=$ $\cos ^{2} \frac{t}{2}(\sin t)^{-1} T_{2 n}^{\alpha_{0}}(\pi-t)$. By a calculation similar to that in the proof of Lemma 6 we obtain

$$
\begin{aligned}
V_{n}(t)> & -a_{1} \sin ^{2} \frac{t}{2}+\sum_{k=1}^{\infty} k a_{2 k-1}-\sum_{k=n+1}^{\infty} k a_{2 k-1} \\
& +\frac{1}{2 \cos t / 2} \sum_{k=1}^{n-1} a_{2 k+1}\left(\sin ^{2} \frac{t}{4}-\sin ^{2}\left(k+\frac{3}{4}\right)\right) \\
& +\frac{n}{2\left(2 n-1+\alpha_{0}\right)\left(2 n+\alpha_{0}\right)}\left(1-\frac{2}{2 n+1+\alpha_{0}}\right) \\
& +\frac{\sin 2 n t}{4\left(2 n-1+\alpha_{0}\right)\left(2 n+\alpha_{0}\right) \sin t}\left(1-\frac{2}{2 n+1+\alpha_{0}}\right) \\
& +\frac{1}{4\left(2 n+\alpha_{0}\right)}-\frac{\sin (2 n+0.5) t}{2\left(2 n+\alpha_{0}\right) 2 \sin t / 2}
\end{aligned}
$$

We write

$$
\begin{aligned}
\delta_{n}= & \frac{n}{2\left(2 n-1+\alpha_{0}\right)\left(2 n+\alpha_{0}\right)}\left(1-\frac{2}{2 n+1+\alpha_{0}}\right) \\
& -\frac{1}{2} \frac{1}{2 n+1+\alpha_{0}}+\frac{1}{4\left(2 n+\alpha_{0}\right)},
\end{aligned}
$$


and note that

$$
\delta_{n}>\frac{0.2225-0.7775(n+0.555)^{-1}}{\left(2 n+\alpha_{0}\right)\left(2 n+1+\alpha_{0}\right)} .
$$

We still use the notation $\Delta_{n}$ defined in Lemma 7, and write also

$$
\begin{aligned}
G_{n}(t)= & F\left(\alpha_{0}\right)-\frac{\sin (2 n+0.5) t}{2\left(2 n+\alpha_{0}\right) 2 \sin \frac{t}{2}}-\frac{1}{2 \cos \frac{t}{2}} \sum_{k=1}^{n-1} a_{2 k+1} \sin ^{2}\left(k+\frac{3}{4}\right) t \\
& +\delta_{n}+\left(1-\frac{2}{2 n+1+\alpha_{0}}\right) \frac{\sin 2 n t}{4\left(2 n-1+\alpha_{0}\right)\left(2 n+\alpha_{0}\right) \sin t} .
\end{aligned}
$$

Then we get

$$
V_{n}(t)>G_{n}(t)+\Delta_{n+1}(t)
$$

LEMMA 14. If $\mu_{0} t /(2 n+0.5) \leq t<\pi /(2 n+0.5), n \geq 3$ then $G_{n}(t)>0$ and $\Delta_{n+1}(t)>0$.

LEMMA 15. If $t=(2 \ell \pi+\delta \pi) /(2 n+0.5)<0.25 \pi, \ell \geq 1$ and $0<\delta<1$, then $V_{n}(t)>0$. In this case, $n \geq 4$ and we have $G_{n}(t)>0.02598$ and $\Delta_{n+1}(t)>$ -0.00452 .

A combination of Lemmas 13-15 together with a direct check of low order cases completes the proof of Theorem 2 .

\section{Acknowledgement}

This work was supported by a grant from the Australian Research Council.

\section{References}

[1] R. Askey and G. Gasper, 'Inequalities for Polynomials', in: The Bieberbach conjecture, Math. Surveys Monographs 21 (Amer. Math. Soc., Providence, 1986) pp. 7-32.

[2] G. Brown and D. C. Wilson, 'A class of positive trigonometric sums II', Math. Ann. 285 (1989), 57-74.

[3] L. Fejér, 'Einige Sätze, die sich auf das Vorzeichen einer ganzen rationalen Funktion beziehen', Monats. Math. Phys. 35 (1928), 305-344.

[4] G. Gasper, 'Nonnegative sums of cosine, ultraspherical and Jacobi polynomials', J. Math. Anal. Appl. 26 (1969), 60-68.

[5] W. Rogosinski and G. Szegö, 'Über die Abschnitte von Potenzreihen, die in einem Kreise beschränkt bleiben', Math. Z. 28 (1928), 73-94. 
[6] W. H. Young, 'On a certain series of Fourier', Proc. London Math. Soc. 11 (1912), 357-366.

Department of Mathematics

The University of Adelaide

Adelaide SA 5005

Australia
Department of Mathematics

Beijing Normal University

Beijing 100875

China 